\title{
BMJ Open New clinical decision rule to exclude subarachnoid haemorrhage for acute headache: a prospective multicentre observational study
}

\author{
Akio Kimura, ${ }^{1}$ Kentaro Kobayashi, ${ }^{1}$ Hitoshi Yamaguchi, ${ }^{2}$ Takeshi Takahashi, ${ }^{3}$ \\ Masahiro Harada, ${ }^{3}$ Hideki Honda, ${ }^{4}$ Yoshio Mori, ${ }^{5}$ Keika Hirose, ${ }^{1}$ Noriko Tanaka ${ }^{6}$
}

To cite: Kimura $A$ Kobayashi K, Yamaguchi $\mathrm{H}$, et al. New clinical decision rule to exclude subarachnoid haemorrhage for acute headache: a prospective multicentre observational study. BMJ Open 2016;6: e010999. doi:10.1136/ bmjopen-2015-010999

- Prepublication history for this paper is available online. To view these files please visit the journal online (http://dx.doi.org/10.1136/ bmjopen-2015-010999).

Received 30 December 2015 Revised 13 July 2016 Accepted 19 August 2016

CrossMark

For numbered affiliations see end of article.

Correspondence to

Dr Akio Kimura;

akimura@hosp.ncgm.go.jp

\section{ABSTRACT}

Objective: To ensure good outcomes in the management of subarachnoid haemorrhage (SAH), accurate prediction is crucial for initial assessment of patients presenting with acute headache. We conducted this study to develop a new clinical decision rule using only objectively measurable predictors to exclude SAH, offering higher specificity than the previous Ottawa SAH Rule while maintaining comparable sensitivity.

Design: Multicentre prospective cohort study.

Setting: Tertiary-care emergency departments of five general hospitals in Japan from April 2011 to March 2014.

Participants: Eligible patients comprised 1781 patients aged $>15$ years with acute headache, excluding trauma or toxic causes and patients who presented in an unconscious state.

Main outcome measures: Definitive diagnosis of SAH was based on confirmation of SAH on head CT or lumbar puncture findings of non-traumatic red blood cells or xanthochromia.

Results: A total of 1561 patients were enrolled in this study, of whom 277 showed SAH. Using these enrolled patients, we reached a rule with mainly categorical predictors used in previous reports, called the 'Ottawalike rule', offering $100 \%$ sensitivity when using any of age $\geq 40$ years, neck pain or stiffness, altered level of consciousness or onset during exertion. Using the 1317 patients from whom blood samples were obtained, a new rule using any of systolic blood pressure $>150 \mathrm{~mm} \mathrm{Hg}$, diastolic blood pressure $>90 \mathrm{~mm} \mathrm{Hg}$, blood sugar $>115 \mathrm{mg} / \mathrm{dL}$ or serum potassium $<3.9 \mathrm{mEq} / \mathrm{L}$ offered $100 \%$ sensitivity $(95 \%$ Cl $98.6 \%$ to $100 \%$ ) and $14.5 \%$ specificity $(12.5 \%$ to $16.9 \%$ ), while the Ottawa-like rule showed the same sensitivity with a lower specificity of $8.8 \%(7.2 \%$ to $10.7 \%$ ).

Conclusions: While maintaining equal sensitivity, our new rule seemed to offer higher specificity than the previous rules proposed by the Ottawa group. Despite the need for blood sampling, this method can reduce unnecessary head CT in patients with acute headache. Trial registration number: UMIN 00004871.

\section{Strengths and limitations of this study}

- In this multicentre cohort study, we developed a new clinical decision rule to exclude subarachnoid haemorrhage (SAH) (Emergency Medicine, Registry Analysis, Learning and Diagnosis, EMERALD SAH Rule) in patients presenting with acute headache at the emergency department in Japan. We selected objectively measurable predictors (systolic and diastolic blood pressures and blood sugar and serum potassium) having no interobserver differences. Maintaining 100\% sensitivity, the EMERALD SAH rule could show higher specificity than the previous rules.

- The proposed rule needs to be externally validated before being fully incorporated into clinical practice, because we only undertook bootstrapping analysis for internal validation.

- Careful application of the EMERALD SAH rule to emergency departments with a low prevalence of SAH appears warranted.

\section{INTRODUCTION}

Subarachnoid haemorrhage (SAH) is a common, serious problem encountered in emergency departments (EDs), and has been reported to result in disability or even death in $40-60 \%$ of affected patients. ${ }^{1-4}$ Good outcomes are strongly dependent on prompt diagnosis and early treatment, ${ }^{1-4}$ while untreated patients can experience sudden clinical deterioration because of rebleeding. 'Sudden, worst headache of life' or 'thunderclap headache' are widely accepted predictors of SAH, and most emergency physicians investigate patients with such characteristic headaches using head CT or lumbar puncture. However, some patients with SAH do not present with such characteristic headaches and $12 \%$ of cases are reportedly overlooked on initial assessment. ${ }^{1-5}$ 
Moreover, a sudden, severe headache can also be found in patients with a more benign headache. ${ }^{6}$ Overlooking $\mathrm{SAH}$ in an alert patient can lead to catastrophic disability or death, ${ }^{5}$ so the development of methods for clinical prediction with high sensitivity is very important, particularly for patients with uncharacteristic symptoms.

The research group at the University of Ottawa has provided highly sensitive clinical decision rules to exclude SAH in patients presenting with acute headache. ${ }^{7}$ Their well-organised research led to a rule including any patient aged $\geq 40$ with neck pain or stiffness, witnessed loss of consciousness or onset during exertion. However, a multicentre cohort validation study failed to show $100 \%$ sensitivity. ${ }^{8}$ The group then added 'thunderclap headache' and 'limited neck flexion on examination', resulting in $100 \%$ sensitivity for the Ottawa SAH Rule. $^{8}$

Over the past decade, we have been involved in the development of clinical predictions to exclude $\mathrm{SAH}^{9} 10$ for patients presenting to EDs with acute headache. Several years ago, we developed the subarachnoid haemorrhage prediction score (SPS) ${ }^{10}$ using only measurable predictors (systolic blood pressure, blood sugar, serum potassium and white cell count) in order to minimise interobserver differences and observer biases. The SPS offered $100 \%$ sensitivity for predicting $\mathrm{SAH}$ in a retrospective single-centre study, but recent prospective validation cohorts have failed to maintain $100 \%$ sensitivity (unpublished data).

The present study was conducted as part of the Emergency Medicine, Registry Analysis, Learning and Diagnosis (EMERALD) project, which is aimed at minimising life-threatening diseases being overlooked at EDs in Japan. The objective of this study was to develop a new clinical decision rule using only objectively measurable predictors to exclude SAH, while maintaining $100 \%$ sensitivity and offering higher specificity than the Ottawa SAH rule. Our new rule may need blood sampling, but was aimed at further reducing unnecessary CT and lumbar puncture, thus limiting costs, exposure to radiation and invasiveness.

\section{METHODS}

\section{Study design}

This prospective multicentre observational study was conducted through the EDs of five general hospitals in Japan from April 2011 to March 2014. The research ethics board at each participating hospital approved the study protocol, which was designed in accordance with the STROBE statement for observational studies.

\section{Study population}

A total of 1899 patients aged $>15$ years with a chief symptom of acute headache and presenting within 14 days of onset were considered for enrolment. We excluded patients with headache caused by trauma, drugs or alcohol, and those who were unconscious at the beginning of assessment. As with previous studies, ${ }^{78}$ we also excluded patients with recurrent headache syndromes (history of $\geq 3$ recurrences of headache with the same characteristics and intensity as the presenting headache over a period $>6$ months).

\section{Data collection}

All patient assessments were made by residents supervised by staff physicians or attending emergency physicians. Physicians were oriented to the study and instructed to input clinical findings at the time of assessment into data collection software specially developed by the EMERALD project on a smartphone, or onto electronic charts of a hospital that showed the same data items as the smartphone device. Electronic chart data were later manually transferred to the smartphone device.

To minimise interobserver differences and observer biases, as in our previous study, we focused on objectively measurable data such as age, heart rate, systolic and diastolic blood pressures and body temperature, which were defined as the first reading by the treating nursing staff. We also collected a variety of data from blood samples, such as blood sugar, serum sodium, serum potassium, haemoglobin concentration, white cell counts and platelet counts, as these factors need only a small amount of blood to determine. In other words only routine examination items for emergency patients in Japanese EDs were used and all results were obtainable within $10 \mathrm{~min}$.

All patient data were anonymised before being uploaded to the internet server via direct smartphone connection or from personal computers at EDs with Bluetooth connections to smartphone devices. Collected anonymised data were monitored and cleaned by the Joint Center for Researchers, Associates and Clinicians (JCRAC), an authorised centre for quality management of data. The final data set for analyses was provided by JCRAC.

\section{Outcome measure}

The primary outcome, SAH, was defined as any of the following: SAH on unenhanced CT of the head; xanthochromia in cerebrospinal fluid; or non-traumatic, bloody cerebrospinal fluid in the final tube sample at lumbar puncture (LP) followed by either angiography or CT angiography to confirm whether an underlying pathology is causing $\mathrm{SAH}$, in cooperation with a neurological staff (neurologist or neurosurgeon) and emergency physicians. All participating hospitals had 64-row, multidetector row (MD) CT scanners either within the ED or located nearby. CT is available within 1 hour, even at midnight. Even if results seemed negative on plain CT, LP was only performed for those patients showing equivocal results on subsequent MRI or CT angiography, but for whom a high index of suspicion for SAH remained. Radiologists interpreted CT images later, and provided a final radiology report. Radiologists checked CT misinterpretations of negative SAH. When 
radiologists found a very subtle SAH on CT, they contacted the ED. If emergency physicians recognised a mistake after discharge, they contacted the patient to come back to the ED as soon as possible. Discharged patients were evaluated by outpatient follow-up or by telephone interview. A total of 188 discharged patients without either CT images or follow-up evaluations were excluded, as their primary outcomes could not be confirmed.

\section{Data analysis}

Univariate analyses were used to determine the strength of association between each possible predictor variable and the outcome variable. We used a two-sided t-test for continuous variables and Fisher's exact test for categorical variables.

To develop the clinical decision rule, we followed previously established methodological standards. ${ }^{11}$ First, we selected categorical variables showing values of $\mathrm{p}<0.05$ on

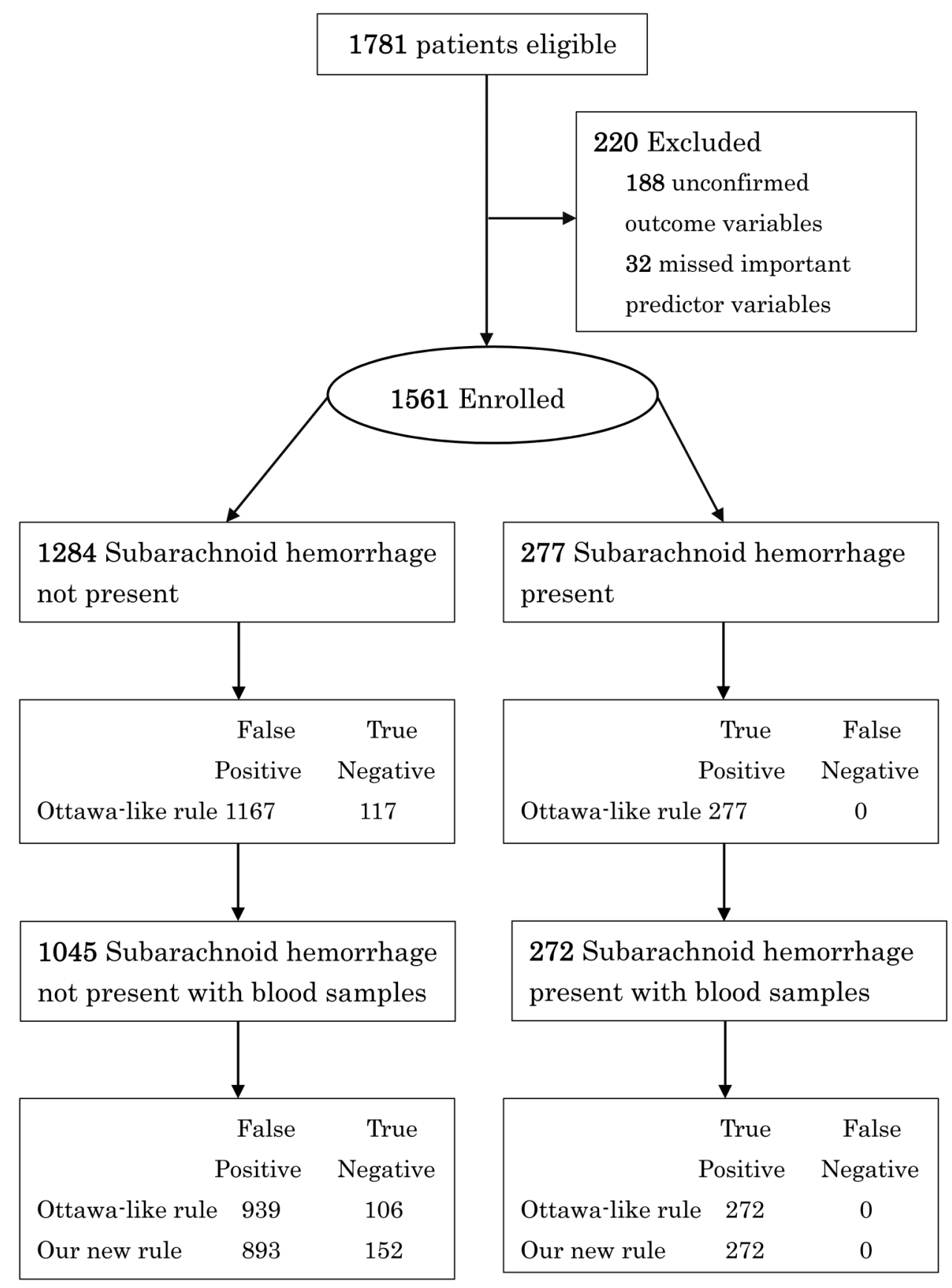

Figure 1 Details of enrolment and flow of patients in study Ottawa-like rule: any of the following risks present: age $\geqq 40$ years; neck pain or stiffness; altered level of consciousness; onset during exertion. Our new rule (namely, the EMERALD SAH rule): any of the following risks present: systolic blood pressure $>150 \mathrm{~mm} \mathrm{Hg}$; diastolic blood pressure $>90 \mathrm{~mm} \mathrm{Hg}$; blood sugar $>115 \mathrm{mg} / \mathrm{dL}$ (6.9 mmol/L); serum potassium $<3.9 \mathrm{mEq} / \mathrm{L}$ (3.90 mmol/L). EMERALD, Emergency Medicine, Registry Analysis, Learning and Diagnosis; $\mathrm{SAH}$, subarachnoid haemorrhage. 
univariate analyses. Then we selected possible predictors as clinically important, continuous variables showing values of $p<0.05$ on univariate analyses. The reason we used objectively measurable variables was mentioned earlier. Setting the presence (1) or absence $(0)$ of SAH as the outcome variable, we performed multivariate, recursive partitioning analyses to develop rules using only the selected categorical variables and age and using only the selected, objectively measurable predictors. Cut-offs for variables were determined in the process of recursive partitioning. Sensitivity and specificity were estimated for each rule. A clinical decision rule for a life-threatening event like SAH requires $100 \%$ sensitivity with a narrow CI. On the basis of this philosophy, we selected the practical new rule with the highest specificity.

We conducted bootstrapping analysis of 1000 iterations to determine the internal stability of rules, and then calculated the sensitivity and specificity of them. Analyses were performed using JMP V.11.2.0 (SAS Institute, Cary, North Carolina, USA) and SAS V.9.3M2 (SAS Institute).

The funding source played no role in the collection, analysis or interpretation of data, the writing of the report or the decision to publish.

\section{RESULTS}

Applied the exclusion criteria to the 1899 consecutive patients, the study flow for the 1781 eligible patients is shown in figure 1 . Of these, 1561 patients $(87.6 \%)$ were enrolled after excluding 220 patients, of whom 188 had unconfirmed outcome variables as mentioned in the Methods and 32 were missing important information about age, onset, neck pain or stiffness, or alteration of level of consciousness. Blood test results were available for 1317 patients $(84.4 \%)$.

Table 1 reports characteristics of the enrolled patients (mean age, 53 years; $58.4 \%$ women), including 277 patients $(17.7 \%)$ with SAH. CT scans were performed for $94.4 \%$ of the enrolled patients, while lumbar punctures were carried out for 2.6\%; $64.7 \%$ of the enrolled patients were discharged from the ED.

Characteristics of the 1781 eligible patients were similar to those of enrolled patients (mean age, 53 years; $58.6 \%$ women). However, 188 patients who were not followed up tended to be younger (mean age 42 years) and to have lower frequencies of both onset during exertion $(15 \%)$ and alteration of consciousness $(2 \%)$. Also, their systolic blood pressure (SBP) and diastolic blood pressure (DBP) tended to be low $(130 \mathrm{~mm} \mathrm{Hg}$ and $78 \mathrm{~mm} \mathrm{Hg}$, respectively).

Table 2 shows the results of univariate analysis. Patients with SAH were older and more often showed onset during exertion, 'worst headache of life', altered level of consciousness, neck pain or stiffness, vomiting and history of hypertension. SBP and DBP were higher in patients with SAH, but surface body temperature was
Table 1 Characteristics of enrolled patients $(\mathrm{N}=1561)$

\begin{tabular}{|c|c|c|}
\hline Characteristics & \multicolumn{2}{|c|}{ Patients } \\
\hline Age, mean (SD) (range) & \multicolumn{2}{|c|}{$\begin{array}{l}53(21) \\
(16-98)\end{array}$} \\
\hline Women & 912 & $58.4 \%$ \\
\hline Onset during exertion & 312 & $20.0 \%$ \\
\hline Onset during rest & 1131 & $72.5 \%$ \\
\hline Headache awoke patient from sleep & 55 & $3.5 \%$ \\
\hline \multicolumn{3}{|l|}{ Duration from onset } \\
\hline$\sim 60 \mathrm{~min}$ & 297 & $19.0 \%$ \\
\hline 24 hours & 863 & $55.3 \%$ \\
\hline$\sim 7$ days & 342 & $21.9 \%$ \\
\hline 1 week & 58 & $3.7 \%$ \\
\hline Worst headache of life & 274 & $17.6 \%$ \\
\hline Thunderclap headache & 37 & $2.4 \%$ \\
\hline Alteration of consciousness level & 151 & $9.7 \%$ \\
\hline Neck pain or stiffness & 1095 & $70.1 \%$ \\
\hline Vomiting & 442 & $28.3 \%$ \\
\hline Vertigo/dizziness & 206 & $13.2 \%$ \\
\hline History of hypertension & 374 & $24.0 \%$ \\
\hline History of diabetes mellitus & 118 & $7.6 \%$ \\
\hline Heart rate, mean $(\mathrm{SD})$ beats/min & 79 & $(17)$ \\
\hline \multicolumn{3}{|l|}{ Blood pressure, mean (SD) $\mathrm{mm} \mathrm{Hg}$} \\
\hline Systolic & 144 & (33) \\
\hline Diastolic & 83 & (19) \\
\hline Body temperature, mean (SD) ${ }^{\circ} \mathrm{C}$ & 36.6 & $(0.8)$ \\
\hline \multicolumn{3}{|l|}{ Diagnostic procedures } \\
\hline CT & 1474 & $94.4 \%$ \\
\hline MRI & 66 & $4.2 \%$ \\
\hline Lumbar puncture & 40 & $2.6 \%$ \\
\hline No CT, lumbar puncture or MRI & 87 & $5.6 \%$ \\
\hline $\begin{array}{l}\text { Discharged from emergency department } \\
\text { Final diaanosis }\end{array}$ & 1010 & $64.7 \%$ \\
\hline Cerebrovascular disease (CVD) & 369 & $23.6 \%$ \\
\hline Subarachnoid haemorrhage & 277 & $17.7 \%$ \\
\hline Other CVD & 92 & $5.9 \%$ \\
\hline Other neurological disease & 715 & $45.8 \%$ \\
\hline Migraine headache & 133 & $8.5 \%$ \\
\hline Tension headache & 61 & $3.9 \%$ \\
\hline Cluster headache & 12 & $0.8 \%$ \\
\hline Unclassified benign headache & 438 & $28.1 \%$ \\
\hline Meningitis & 17 & $1.1 \%$ \\
\hline Postseizure headache & 15 & $1.0 \%$ \\
\hline Neuralgia & 10 & $0.6 \%$ \\
\hline Brain tumour & 7 & $0.4 \%$ \\
\hline Viral illness & 60 & $3.8 \%$ \\
\hline Psychiatric disease & 47 & $3.0 \%$ \\
\hline Hypertensive crisis & 38 & $2.4 \%$ \\
\hline Peripheral vertigo & 37 & $2.4 \%$ \\
\hline Gastrointestinal disease & 22 & $1.4 \%$ \\
\hline Sinusitis & 16 & $1.0 \%$ \\
\hline Hyperventilation & 16 & $1.0 \%$ \\
\hline Urinary tract infection & 16 & $1.0 \%$ \\
\hline Dehydration & 15 & $1.0 \%$ \\
\hline Respiratory disease & 14 & $0.9 \%$ \\
\hline Syncope & 10 & $0.6 \%$ \\
\hline Cervical spondylosis & 10 & $0.6 \%$ \\
\hline Other non-neurological disease & 176 & $11.3 \%$ \\
\hline
\end{tabular}


Table 2 Univariate correlation of variables for subarachnoid haemorrhage

\begin{tabular}{|c|c|c|c|}
\hline \multirow[b]{2}{*}{ Characteristic } & \multicolumn{2}{|c|}{ Subarachnoid haemorrhage } & \multirow[b]{2}{*}{ p Value } \\
\hline & No $(n=1284)$ & Yes $(n=277)$ & \\
\hline \multicolumn{4}{|l|}{ From history } \\
\hline Age, mean (SD) & $51(21)$ & $63(15)$ & $<0.0001$ \\
\hline Women & $56.9 \%$ & $67.5 \%$ & 0.0012 \\
\hline Onset during exertion & $14.3 \%$ & $54.9 \%$ & $<0.0001$ \\
\hline Worst headache of life & $9.5 \%$ & $54.9 \%$ & $<0.0001$ \\
\hline Thunderclap headache & $2.2 \%$ & $3.3 \%$ & 0.279 \\
\hline Altered level of consciousness & $3.8 \%$ & $40.1 \%$ & $<0.0001$ \\
\hline Neck pain or stiffness & $72.9 \%$ & $86.6 \%$ & $<0.0001$ \\
\hline Vomiting & $25.4 \%$ & $52.7 \%$ & $<0.0001$ \\
\hline Vertigo/dizziness & $15.6 \%$ & $8.3 \%$ & 0.0072 \\
\hline History of hypertension & $24.0 \%$ & $43.7 \%$ & $<0.0001$ \\
\hline History of diabetes mellitus & $10.1 \%$ & $3.6 \%$ & 0.095 \\
\hline \multicolumn{4}{|l|}{ From physical examination } \\
\hline Heart rate, mean (SD) beats/min & 79 (17) & $80(17)$ & 0.7669 \\
\hline \multicolumn{4}{|l|}{ Blood pressure, mean (SD) $\mathrm{mm} \mathrm{Hg}$} \\
\hline Systolic & $139(30)$ & $167(36)$ & $<0.0001$ \\
\hline Diastolic & $81(18)$ & $93(21)$ & $<0.0001$ \\
\hline Body temperature, mean (SD) ${ }^{\circ} \mathrm{C}$ & $36.6(0.8)$ & $36.3(0.9)$ & $<0.0001$ \\
\hline \multicolumn{4}{|l|}{ Diagnostic procedures } \\
\hline $\mathrm{CT}$ & 1197 & 277 & \\
\hline MRI & 62 & 4 & \\
\hline Lumbar puncture & 37 & 3 & \\
\hline From blood test & No $(n=1045)$ & Yes $(n=272)$ & \\
\hline Blood sugar, mean (SD) mg/dL & $127(51)$ & $162(49)$ & $<0.0001$ \\
\hline Serum sodium, mean (SD) $\mathrm{mEq} / \mathrm{L}$ & $139.4(3.4)$ & $138.6(3.0)$ & 0.0016 \\
\hline Serum potassium, mean (SD) $\mathrm{mEq} / \mathrm{L}$ & $3.9(0.5)$ & $3.6(0.5)$ & $<0.0001$ \\
\hline Haemoglobin, mean (SD) g/dL & $13.6(2.0)$ & $13.4(1.9)$ & 0.1044 \\
\hline White cell count, mean (SD) $\times 10^{3} / \mu \mathrm{L}$ & $7.9(3.4)$ & $10.3(4.6)$ & $<0.0001$ \\
\hline Platelet count, mean (SD) $\times 10^{4} / \mu \mathrm{L}$ & $21.7(10.0)$ & $24.0(21.0)$ & 0.9258 \\
\hline
\end{tabular}

lower. In the 1317 patients for whom blood test results were available, blood sugar level and white cell count were higher in patients with $\mathrm{SAH}$, but serum potassium was slightly lower.

From the 1561 enrolled patients, as a result of recursive partitioning analysis, we developed almost the same rule as Rule 1 from the previous literature, ${ }^{7}$ using any of age $\geq 40$ years, neck pain or stiffness, altered level of consciousness or onset during exertion. This rule was termed the 'Ottawa-like rule', showing 100\% sensitivity (95\% CI 98.6\% to $100 \%$ ) and $9.1 \%$ specificity (95\% CI $7.7 \%$ to $10.8 \%$ ).

Using data from the 1317 patients for whom blood results were available, we developed a new rule using any of SBP >150 $\mathrm{mm} \mathrm{Hg}$, DBP $>90 \mathrm{~mm} \mathrm{Hg}$, blood sugar $>115 \mathrm{mg} / \mathrm{dL} \quad(6.9 \mathrm{mmol} / \mathrm{L})$ or serum potassium $<3.9 \mathrm{mEq} / \mathrm{L} \quad(3.9 \mathrm{mmol} / \mathrm{L})$ (figure 2). This new rule, which we called the 'EMERALD SAH rule', offered $100 \%$ sensitivity $(95 \%$ CI $98.6 \%$ to $100 \%)$ and $14.5 \%$ specificity (95\% CI $12.5 \%$ to $16.9 \%$ ). In comparison, the Ottawa-like rule showed identical sensitivity, but a lower specificity of $8.8 \%$ (95\% CI $7.2 \%$ to $10.7 \%)$.

Ninety of the other 92 patients with cerebrovascular disease (table 1) met the criteria of the EMERALD rule. A tiny subcortical haemorrhage and thalamic haemorrhage $1-2 \mathrm{~cm}$ in diameter occurred in two patients.

\section{DISCUSSION}

Over the past decade, we have been looking for rigid measurable predictors that would leave no room for interobserver disagreement. We have identified age, blood pressures, body temperature, blood sugar concentration, serum potassium concentration and white cell count as possible predictors (table 2). For the derivation of our new EMERALD SAH Rule, we selected SBP and DBP and blood sugar and serum potassium levels, as these offered relatively stronger discriminant abilities. We excluded age as a factor already used in the Ottawa SAH Rule.

Our data demonstrated $100 \%$ sensitivity for the Ottawa-like rule, almost identical to Rule 1 ,which has the highest sensitivity among the three rules developed by the Ottawa group. ${ }^{7}$ Prospective validation was provided for those three rules, ${ }^{8}$ showing that Rule 1 had the highest sensitivity of $98.5 \%$ and $27.5 \%$ specificity. However, $100 \%$ sensitivity was not achieved. From this result, the Ottawa group proposed the Ottawa SAH Rule $^{8}$ with $100 \%$ sensitivity, adding 'thunderclap headache' and 'limited neck flexion', despite making the specificity $12.2 \%$ lower than that of Rule 1 . This represented an even lower specificity than the EMERALD SAH Rule in our study. 
Figure 2 Example of recursive partitioning analysis with our new rule: the EMERALD SAH Rule. EMERALD, Emergency Medicine, Registry Analysis, Learning and Diagnosis; SAH, subarachnoid haemorrhage.

\section{Systolic blood pressure $>150 \mathbf{~ m m H g}$ ?}

1317 patients, 272 had SAH

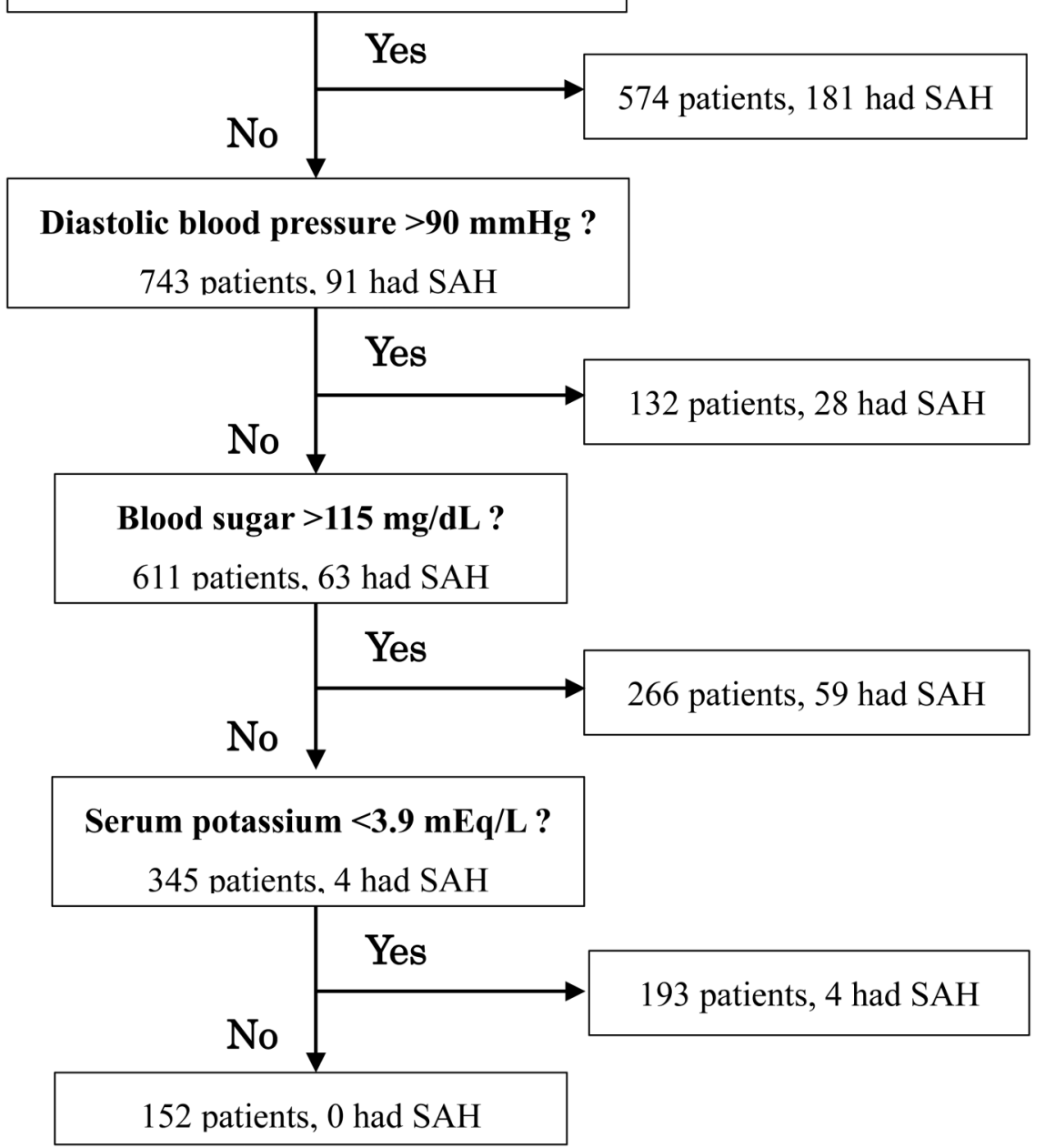

We thus proposed a two-step decision-making rule (figure 3). We placed the Ottawa SAH Rule as the first screening step, because that is much more clinically intuitive and needle puncture is not needed. We can exclude SAH without unnecessary blood sampling for some patients with acute headache. However, if the EMERALD SAH Rule is placed as the second step, we can obtain higher specificity without reducing the optimal sensitivity, thus hopefully reducing both unnecessary exposure to radiation and costs from CT, while requiring only the small blood volume needed for blood gas analysis, and also reducing the need for invasive LPs that might result in a headache even worse than the presenting symptom.

Several studies have found hypertension to represent an independent risk factor for SAH. ${ }^{4}$ Before this study, high SBP and DBP had already been selected as possible predictors with similar cut-off values by the Canadian group, ${ }^{7}$ but were not chosen as final predictors for the Ottawa SAH Rule.

We did not find any studies directly investigating the correlation between elevated blood sugar levels and
SAH but, according to Douhout and colleagues, ${ }^{12}$ higher blood sugar levels in the first 10 days after SAH are associated with worsened outcomes. Frontera $e t a l^{13}$ have also claimed that SAH is generally followed by hyperglycaemia, suggesting some correlation between the development of SAH and elevated blood sugar levels. A recent meta-analysis ${ }^{14}$ revealed that admission glucose levels are often high and hyperglycaemia is associated with an increased risk of poor outcomes after SAH.

Serum potassium levels were also significantly decreased in patients with SAH. SAH is believed to result in accelerated catecholamine secretion and increased intracellular potassium uptake, leading to lower serum potassium levels. ${ }^{15-17}$

\section{Limitations}

Our study cohort contained a higher proportion (17.7\%) of positive SAH than the Ottawa SAH Rule cohort. One contributor to the high rate of SAH in this study may have been the higher percentage of patients referred from smaller hospitals or clinics to EDs of 
Figure 3 The proposed two-step

decision-making to rule out subarachnoid haemorrhage (SAH) for adult patients with acute headache.

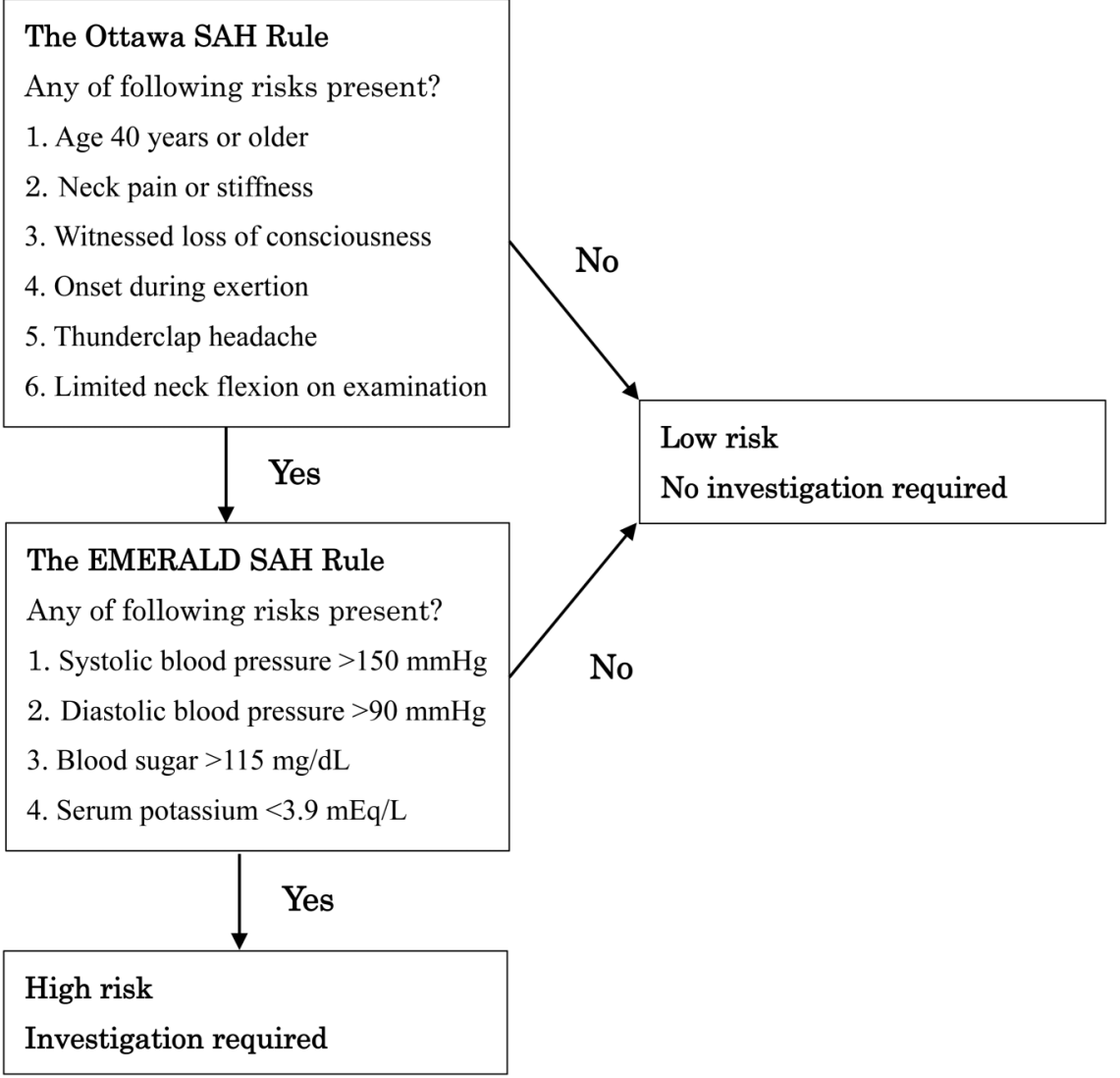

tertiary general hospitals, like those participating in this study in Japan, compared to those in EDs of hospitals in North America. Walk-in patients with headaches may attend smaller, secondary hospitals or clinics. Patients with a strong index of suspicion for SAH may be taken by ambulance to bigger general hospitals.

Another issue warranting attention was the fact that the LP rate was very low at $2.6 \%$. If results from noncontrast CT are negative, LP follow-up seems to represent a standard of care. ${ }^{18}$ However, LP is very invasive, requires patient cooperation and might cause further headache. Among member countries of the organisation for Economic Co-operation and Development, Japan has the largest number of CT scanners per million people and the same situation can also be seen in the number of MRI scanners. Easy access to fifth-generation or later imaging by MDCT, which seems to detect SAH with sensitivity approaching $100 \%,{ }^{19}$ are routinely assured at all hospitals participating in this study, as well as at almost every general hospital in Japan. This allows CT to be obtained within 1 hour from arrival in the ED. Moreover, easy access to EDs is provided all over Japan, so most patients with acute headache will present to hospitals within a few hours of onset. Among patients with negative results on non-contrast CT, as confirmed by emergency physicians, neurological doctors and radiologists, but still showing a high suspicion of SAH, Japanese emergency physicians prefer to perform the less invasive and more specific CT angiography ${ }^{20}$ or $\mathrm{MRI}^{2122}$ before
LP, and LP is indicated only if the results of imaging are equivocal. The costs of radiological examinations are covered by the National Health Insurance system of Japan. These were considered the major reasons for the very low frequency of LP.

Although we have explained the Japanese situation for the higher proportion of SAH and low LP rate, the findings might imply that some headaches at risk of representing SAH were not being captured by this study. Careful application of our results to EDs with a lower prevalence of SAH appears warranted.

To minimise observer biases, as we mentioned before, we have focused on objectively measurable data that do not require assessments with a $\kappa$ coefficient. We did not assess interobserver agreement for non-numerical variables, so our results for the Ottawa-like rule carried a risk of interobserver differences. However, the results were not far from those of the Ottawa studies, and may be sufficient to show the superior specificity of the EMERALD SAH Rule using only four measurable variables.

We were unable to record the time to peak headache intensity for all patients, and thus did not know whether headache reached maximum intensity within 1 hour for all patients. This was because patients with non-typical, relatively lighter headache experienced difficulty answering when peak intensity occurred. Moreover, many patients with acute headache are often very reluctant to answer all clinical questions from physicians. This is 
another reason why we chose to rely on objectively measurable findings. The EMERALD SAH Rule would be applicable regardless of the time to peak intensity.

If patients develop new neurological deficits or have a history of aneurysm or brain tumour, precise investigations including non-enhanced and enhanced CT and routine blood testing are conducted in EDs in Japan. We therefore do not emphasise the exclusion of such patients, because we can confirm the presence or absence of SAH. Our data could possibly have included a very small number of such patients. However, the negative effects of this possibility were considered to be almost zero for the derivation of the EMERALD SAH Rule.

The proposed rule needs to be properly validated before being fully incorporated into clinical practice, because we only undertook bootstrapping analysis for internal validation. This rule thus requires external validation before implementation.

In conclusion, despite the necessity of blood testing, our EMERALD SAH Rule shows higher specificity than the previous Ottawa SAH Rule while maintaining equal sensitivity. It can allow further reduction of unnecessary investigations such as CT or LP in patients showing one or more of the predictors of the Ottawa SAH Rule. The EMERALD SAH Rule can play a role as a secondary screening to the Ottawa SAH Rule for further exclusion of SAH in patients with acute headache. However, further validation studies providing comparable results are required before making alterations to clinical practice.

\section{Author affiliations}

${ }^{1}$ Department of Emergency Medicine and Critical Care, Center Hospital of the National Center for Global Health and Medicine, Shinjuku, Tokyo, Japan ${ }^{2}$ Department of Intensive Care Medicine, Ogaki Municipal Hospital, Ogaki City, Gifu, Japan

${ }^{3}$ Department of Emergency and Critical Care, National Hospital Organization Kumamoto Medical Center, Kumamoto City, Kumamoto, Japan

${ }^{4}$ Department of Emergency and Critical Care Medicine, Yokosuka General Hospital Uwamachi, Yokosuka City, Kanagawa, Japan

${ }^{5}$ Emergency and Critical Care Center, Gifu Prefectural General Medical Center, Gifu City, Gifu, Japan

${ }^{6}$ Biostatistics Section, Department of Clinical Research and Informatics, Clinical Science Center, National Center for Global Health and Medicine, Shinjuku, Tokyo, Japan

Acknowledgements The authors would like to express their sincere gratitude to Dr Takaaki Suzuki, Dr Tatsuki Uemura and other residents who helped with data collection, to the staff of the JCRAC data center who assisted in data management, and to the engineers who developed the systems and software for data collection.

Contributors AK has made major contributions to the conception, design, analysis and interpretation of data in this study. KK has made substantial contributions to the conception of this study. HY, TT, MH, HH, YM and KH have made substantial contributions to the acquisition of data in this study. NT has made substantial contributions to the statistical analysis in this study.

Funding This study was supported by grants (21-123 and 24-114) from the National Center for Global Health and Medicine. No additional data are available.

Competing interests None declared.

Ethics approval The research ethics committee at each participating hospital approved the study protocol.
Provenance and peer review Not commissioned; externally peer reviewed.

Data sharing statement No additional data are available.

Open Access This is an Open Access article distributed in accordance with the Creative Commons Attribution Non Commercial (CC BY-NC 4.0) license, which permits others to distribute, remix, adapt, build upon this work noncommercially, and license their derivative works on different terms, provided the original work is properly cited and the use is non-commercial. See: http:// creativecommons.org/licenses/by-nc/4.0/

\section{REFERENCES}

1. Suarez JI, Tarr RW, Selman WR. Aneurysmal subarachnoid hemorrhage. N Engl J Med 2006;354:387-96.

2. van Gijn J, Kerr RS, Rinkel GJ. Subarachnoid haemorrhage. Lancet 2007;369:306-18.

3. Edlow JA, Malek AM, Ogilvy CS. Aneurysmal subarachnoid hemorrhage: update for emergency physician. J Emerg Med 2008;34:237-51.

4. Connolly ES, Rabinstein AA, Carthuapoma JR, et al. Guidelines for the management of aneurysmal subarachnoid hemorrhage. A guideline for healthcare professionals from the American Heart Association/American Stroke Association. Stroke 2012;43:1711-37.

5. Kowalski RG, Claassen J, Kreiter KT, et al. Initial misdiagnosis and outcome after subarachnoid hemorrhage. JAMA 2004;291:866-9.

6. Linn FH, Rinkel GJ, Algra A, et al. Headache characteristics in subarachnoid haemorrhage and benign thunderclap headache. J Neurol Neurosurg Psychiatr 1998;65:791-3.

7. Perry JJ, Stiell IG, Sivilotti ML, et al. High risk clinical characteristics for subarachnoid haemorrhage in patients with acute headache: prospective cohort study. BMJ 2010;341:c5204.

8. Perry JJ, Stiell IG, Sivilotti ML, et al. Clinical decision rules to rule out subarachnoid haemorrhage for acute headache. JAMA 2013;310:1248-55.

9. Kimura Y, Kimura A, Tomioka J, et al. Early diagnosis of subarachnoid hemorrhage in patients reporting headache at emergency center. JJAAM 2001;12:275-81.

10. Kobayashi K, Kimura A, Hagiwara A, et al. Highly sensitive, subarachnoid hemorrhage prediction score for patients with acute headache. JJAAM 2011;22:305-11.

11. Stiell IG, Wells GA. Methodologic standards for the development of clinical decision rules in emergency medicine. Ann Emerg Med 1999;33:437-47.

12. Dorhout Mees SM, van Dijk GW, Algra A, et al. Glucose levels and outcome after subarachnoid hemorrhage. Neurology 2003;61:1132-3.

13. Frontera JA, Fernandez A, Claassen J, et al. Hyperglycemia after $\mathrm{SAH}$ : predictors, associated complications, and impact on outcome. Stroke 2006;37:199-203.

14. Kruyt ND, Bissels GJ, de Hann RJ, et al. Hyperglycemia and clinical outcome in aneurysmal subarachnoid hemorrhage, a meta-analysis. Stroke 2009;40:e424-30.

15. Fukui $\mathrm{S}$, Otani $\mathrm{N}$, Katoh $\mathrm{H}$, et al. Female gender as a risk factor for hypokalemia and QT prolongation after subarachnoid hemorrhage. Neurology 2002:59:134-6.

16. Fukui $\mathrm{S}$, Katoh $\mathrm{H}$, Tsuzuki $\mathrm{N}$, et al. Multivariate analysis of risk factors for QT prolongation following subarachnoid hemorrhage. Crit Care 2003;7:R7-12.

17. Fukui S, Katoh H, Tsuzuki N, et al. Gender disparities in serum electrolytes levels after subarachnoid hemorrhage. J Clin Neurosci 2004;11:606-9.

18. Perry JJ, Spacek A, Forbes $M$, et al. Is the combination of negative computed tomography result and negative lumbar puncture result sufficient to rule out subarachnoid hemorrhage? Ann Emerg Med 2008;51:707-13.

19. Boesiger BM, Schiber JR. Subarachnoid hemorrhage diagnosis by computed tomography and lumber puncture: are fifth generation CT scanners better at identifying subarachnoid hemorrhage? J Emerg Med 2005;29:23-7.

20. McCormack RF, Huston A. Can computed tomography angiography of the brain replace lumbar puncture in the evaluation of acute-onset headache after a negative noncontrast cranial computed tomography scan? Acad Emerg Med 2010;17:444-51.

21. Verma RK, Kottke R, Andereggen L, et al. Detecting subarachnoid hemorrhage: comparison of combined FLAIR/SWI versus CT. Europ $J$ Radiol 2013;82:1539-45.

22. Farzad A, Radian B, Oh JS, et al. Emergency diagnosis of subarachnoid hemorrhage: an evidence-based debate. J Emerg Med 2013;44:1045-53. 